\title{
A systematic review of EPDS cultural suitability with Indigenous mothers: a global perspective
}

\author{
Ai Wen Chan $^{1}$ (D) $\cdot$ Corinne Reid ${ }^{2,3} \cdot$ Petra Skeffington $^{4} \cdot$ Rhonda Marriott $^{5}$
}

Received: 14 July 2020 / Accepted: 12 October 2020 / Published online: 27 November 2020

(C) The Author(s) 2020

\begin{abstract}
The Edinburgh Postnatal Depression Scale (EPDS) is used extensively as the "gold standard" perinatal depression and anxiety screening tool. This study contributes to an emerging discussion about the tool's shortcomings, specifically around cultural suitability for use with Indigenous women. A systematic search was conducted in ProQuest, PsycINFO, MEDLINE (Web of Science), PubMed, Scopus, Informit, and CINAHL research databases, and grey literature. The quality of the body of evidence was assessed using the NHMRC Level of Evidence framework. Three studies supported the cultural validation of the EPDS with Indigenous groups in Canada $(n=2)$ and the USA $(n=1)$. The remaining eleven Australian studies demonstrated that cultural concerns were suggested by either Indigenous mothers, healthcare professionals (Indigenous and non-Indigenous), or both, though cultural concerns were more weighted from the perspectives of healthcare professionals. The quality of the evidence was not strong, and thus, there is a critical and urgent need for targeted research in this area. This review identified and recommended Indigenous-specific methodologies that can be adopted for more trustworthy, culturally safe, and effective research in this area. Given that the EPDS is currently considered gold standard in routine perinatal mental health screening practice in countries around the world, these findings raise significant concerns. Using culturally relevant research methodologies, such as the use of mixed-methods design, could lay stronger groundwork for further investigation of the broader utility and cultural relevance of the tool.
\end{abstract}

Keywords Indigenous $\cdot$ Edinburgh Postnatal Screening Scale (EPDS) $\cdot$ Systematic review $\cdot$ Perinatal mental health $\cdot$ Acceptability

\section{Introduction}

Symptoms of perinatal depression are sometimes mistaken as typical pregnancy responses (physiological, biological/

\section{Ai Wen Chan}

wenn.chan@gmail.com

Corinne Reid

Corinne.Reid@ed.ac.uk

1 School of Psychology, Exercise Science, Chiropractic \& Counselling, Murdoch University, Perth, Australia

2 Victoria University, Melbourne, Australia

3 Global Health Academy, The University of Edinburgh, Edinburgh, Scotland

4 Psychology, Exercise Science, Chiropractic \& Counselling, Murdoch University, Perth, Australia

5 Ngangk Yira Research Centre for Aboriginal Health and Social Equity, Murdoch University, Perth, Australia hormonal, and emotional). Unrecognised and missed diagnosis can result in repercussions like maternal death and stillbirth (Eastwood et al. 2017; Milgrom and Gemmill 2015; National Institute for Health and Care Excellence 2014). Perinatal depression is also associated with health risks and diminished wellbeing of the mother and her family system, such that stress and depression during and after pregnancy can negatively affect an infant's future growth and development (Cao-Lei et al. 2017; Cook et al. 2018; Gentile 2015, 2017; Goodman 2019).

Globally, Indigenous ${ }^{1}$ women and children typically have worse physical and mental health outcomes compared to nonIndigenous counterparts (Jorm et al. 2012; Lima et al. 2019; Trovato and Romaniuk 2014) and continue to experience inadequate and substandard care and treatment due to marginalisation from mainstream healthcare services (Harfield et al. 2018;

\footnotetext{
${ }^{1}$ This paper uses the term "Indigenous" to describe first inhabitants or first claims of land, territory, or country. This term has gained acceptance to use within an international context as it includes peoples who have experienced similar colonising circumstances (Peters and Mika, 2017).
} 
McIntyre et al. 2017; Minority Rights Group International 2016; World Health Organization (WHO) 2013). The health gap is further perpetuated by intersecting forces of socio-economic and political experiences resulting from European colonisation including intergenerational trauma, racial discrimination, cultural disconnection and suppression (i.e., loss of language), systematic oppression and assimilation, loss of control (i.e., land loss and home dispossession), and limited access to basic adequate services like health, education, and employment (Anderson et al. 2016; Aguiar and Halseth 2015; Jackson Pulver et al. 2010; Brave Heart et al. 2011).

The most widely used screening tool for identifying women who may be at risk for depression after childbirth is the Edinburgh Postnatal Depression Scale (EPDS; Cox et al. 1987). Originally developed and validated with a sample of postnatal women in Scotland, the use of EPDS has gained widespread acceptance and extensive use over the ensuing 30 years evidenced by its ubiquitous translation and validation (Alvarado et al. 2015; Cox et al. 2014; Department of Health, Government of Western Australia 2006; Hewitt et al. 2010; Joshi et al. 2020; Rhee et al. 2018; Shrestha et al. 2016). However, in recent years, there has been emerging discussion around the limitations of the EPDS, including questioning of the cultural suitability and validity when applied in Indigenous or cross-cultural contexts (Black et al. 2018; Gausia et al. 2013; Geia et al. 2013; State of Queensland 2014). A recent systematic review reported a lack of research investigating the effectiveness of the standard EPDS with Indigenous Australian women, providing neither psychometric properties nor qualitative validation of the standard EPDS (Kotz et al. 2020).

Arguments about cross-cultural validity include questions about the relevance of some items, language barriers, and differences in conceptualisations of mental health (Brave Heart et al. 2011; Douglas 2013; Guerin and Guerin 2012; Kozinszky and Dudas 2015; Laungani 2000). Evidence of cross-cultural inconsistency in cut-off scores have been identified as supporting these claims (Matthey et al. 2006). Variable cut-off scores could be attributed to the different definitions of depression based on the diagnostic criteria and systems used (Gibson et al. 2009). It may also reflect cultural variation in the expression, manifestation, and representation of depressive and anxious symptoms (Oates et al. 2004). Irrespective of origin, stark disparities in validated cut-off scores call into question the level of confidence in EPDS as a screening tool, yet clinical practice guidelines continue to be guided by validated cut-off scores to assist in reviewing and monitoring, and providing supportive referral pathways for women (Department of Health 2019; McCabe-Beane et al. 2016). Variable cut-off scores may indicate cultural differences, reinforcing the need for a strong emphasis on cultural context across validation studies. Developing an evidence-informed understanding of applicability of the EPDS within Indigenous contexts is a high priority to ensure access to services and welltargeted service provision in this high-risk population.

The primary aim of this systematic review is to elucidate how culturally suitable the EPDS is and how variations of EPDS implementation have been used with Indigenous populations across the world. To date, no such reviews exist. Reviewing international literature within this context facilitates shared learning and is undertaken with an intention to empower Indigenous communities when faced with engagement in mainstream health systems that may not be designed to best meet their needs.

\section{Methods}

\section{Definitions}

Indigenous There is no universally accepted definition of "Indigenous" as Indigenous groups, nations, and/or tribes have heterogeneous and diverse definitions and preferred names. For the sake of this review, the definition of "Aboriginal" or "Indigenous" include persons or a group of people who are native to and the original inhabitants of the land or country and possess understanding of these natural environments before the arrival of settlers ${ }^{2}$ (United Nations 2014).

Perinatal period The perinatal period includes the time of gestation when a baby is conceived until after the baby is born, with varying definitions of what constitutes the length of time after baby is born (American Psychiatric Association 2013; Australian Institute of Health and Welfare 2014; WHO 2016). A timeframe was not specified in the search.

\section{Screening process}

On 19 January 2020, a combination of search terms (see Table 1) was used to systematically search the following electronic databases: ProQuest, PsycINFO, MEDLINE (Web of Science), PubMed, Scopus, Informit (including Indigenous Collection), and CINAHL. The search term "Edinburgh Postnatal Depression Scale" was not included because this limited the search results. Additional sources like grey literature were included to maximise the coverage of a wider depth and breadth of existing Indigenous health literature (Derrick et al. 2012). At every opportunity, original authors of studies were directly contacted in an attempt to gain access to additional and relevant information, though this strategy was not always successful.

\footnotetext{
${ }^{2}$ This definition is not intended to destroy the heterogeneity and diversity of Indigenous groups and tribes and is not an attempt to overlook preferred names by specific nation or tribes.
} 
Table 1 Search terms

\begin{tabular}{|c|c|}
\hline Indigenous & $\begin{array}{l}\text { Aborigin* OR Indigen* OR "Indigenous people*" OR "ethnic minorit*” OR "ethnic group*" OR } \\
\text { "minorit* group*" OR native OR "native nation*" OR "First Nation*" OR "First people*" OR } \\
\text { "native people*" OR "native population*" OR trib* OR "native trib*" OR "American India*" } \\
\text { OR "Alaska* Native*" OR "Native Alaska*" OR "Native America*" OR "Native Hawaii*" } \\
\text { OR "Plain India*” OR "Torres Strait" OR Metis OR Inuit OR Eskimo OR Aleut OR Yupik OR } \\
\text { Maori OR Sami OR Nenet* OR Komi OR Circassian* OR Polynesian* OR Melanesian* OR } \\
\text { Micronesian* OR Papuan* }\end{array}$ \\
\hline Perinatal & $\begin{array}{l}\text { perinat* OR pregnan* OR antenat* OR prenat* OR postnat* OR prepart* OR postpart* OR } \\
\text { peripart* OR antepart* OR intrapart* OR prepart* OR neonat* OR puerper* OR matern* }\end{array}$ \\
\hline Depression & depress* OR anxi* OR mood* OR "affective disorder" OR "mental health" \\
\hline
\end{tabular}

Studies that met the following criteria were included:

1. The study was undertaken after the EPDS was developed and published in 1987 (Cox et al. 1987).

2. EPDS was a primary focus of measurement.

3. The relationship between Indigenous participants and EPDS scores were discussed in detail.

4. Provided evidence and discussion regarding cultural issues and perspectives towards the EPDS.

5. Cross-cultural approaches, strategies, and/or Indigenous research methodologies were used.

Peer-reviewed studies, intervention studies, observational studies, and case reports published in peer-reviewed journals were considered. Study protocols, editorial commentaries, discussion papers, and other variation of an opinion/review papers were excluded.

After duplicates were extracted, $25 \%$ of those studies were further independently screened by a co-author (PS) to confirm final eligibility of studies. These studies were selected through a random function on Excel to ensure inter-rater reliability was met by two reviewers (Waddington et al. 2012). Agreement between WC and PS was strong, $r=0.939$. Eligible studies that were included are shown in Fig. 1.

\section{Final eligibility of studies}

A total of 14 published articles met inclusion criteria. Each stage of the search and screening processes are shown in the Preferred Reporting Items for Systematic Review and MetaAnalysis (PRISMA) flow diagram (Fig. 1).

\section{Quality appraisal criteria}

Studies were ranked according to the National Health and Medical Research Council (NHMRC) levels of evidence (National Health and Medical Research Council, 2009a, b). To accommodate grey literature, a level of evidence based on expert opinion (NHMRC 2000) was added: "Level V: Evidence obtained from expert opinion from clinicians, authorities and/or reports of expert committees".

\section{Results}

Studies were categorised by country, then ordered by the NHMRC level of evidence ratings (Table 2). Eleven studies were conducted in Australia, two in Canada, and one in the United States of America (USA). All selected studies expressed some degree of cultural concern about the EPDS from either mothers' and/or healthcare professionals' (HCP) point of view.

\section{Assessing quality of body of evidence}

Due to the heterogeneity of research methodologies, interventions, and outcomes in the eligible studies, a quantitative summary measure of the results was not applied. Instead, a descriptive analysis of the selected publications enabled more robust comparisons between studies' findings. The synthesis of evidence was weighed transparently, critically addressed, and reviewed according to the National Health and Medical Research Council (NHMRC) framework (NHMRC 2009b). The following ratings assigned for each component will inform future research and recommendations.

Evidence base The quality of the body of evidence was not high - it was constrained by the lowest Level V studies, with Level III-3 $(n=5 / 14)$ being the highest. A Poor (level D) quality of evidence was determined.

Consistency The majority of studies $(n=10)$ conveyed cultural concerns from Indigenous women and/or healthcare professional (HCP) in relation to the standard EPDS, resulting in a Satisfactory (level C) rating.

Clinical impact Variable results suggested that the standard EPDS was not suitable for Indigenous Australian mothers; hence, a rating of Good (level B) was given as the studies presented strong evidence of clinical relevance (challenging current practice) and potentially positive clinical impact if these findings are taken into account when reviewing the use of measures of postnatal depression for Indigenous contexts. 


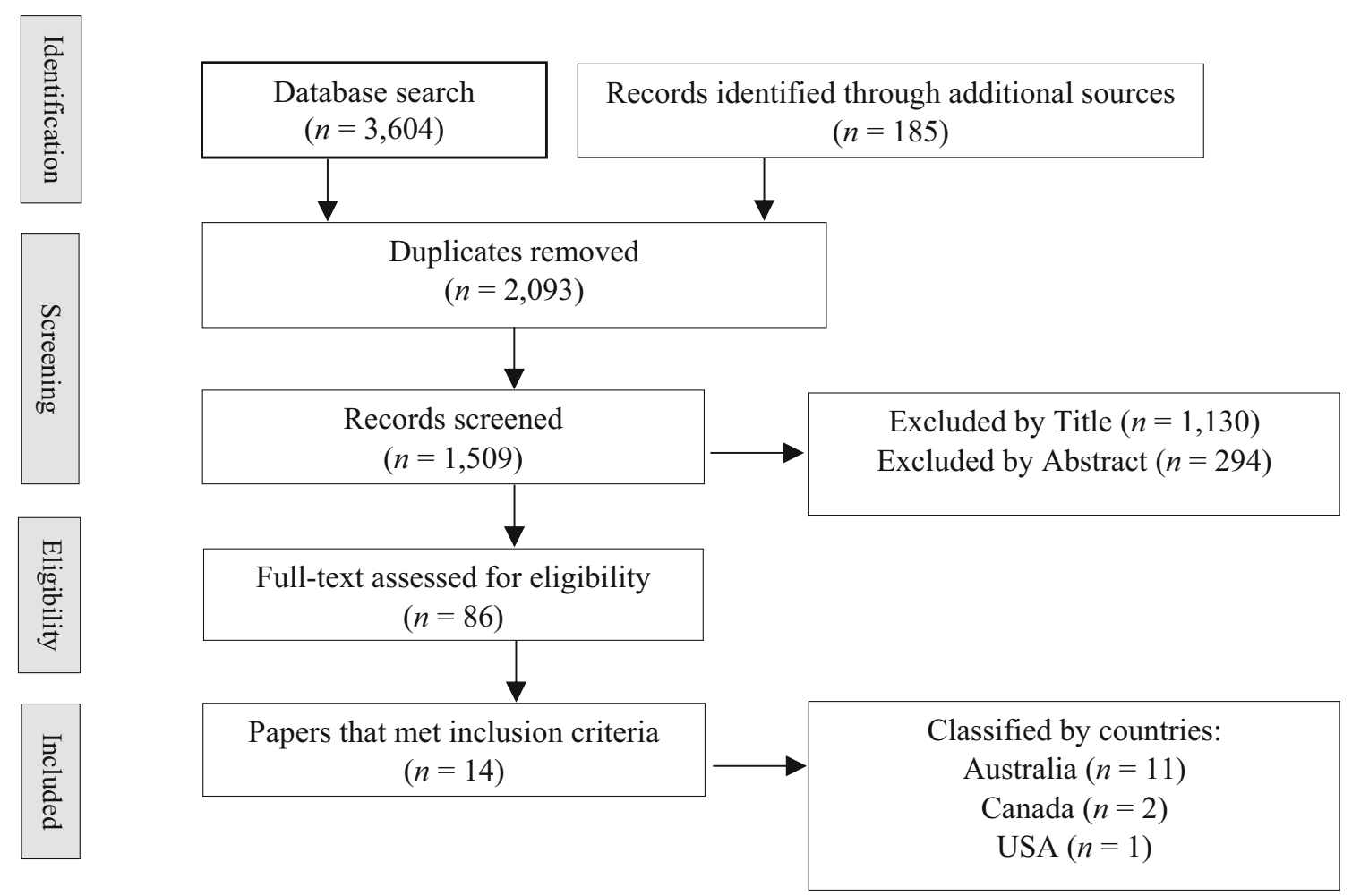

Fig. 1 PRISMA flow chart (Moher et al. 2009) showing identified and screened papers to be assessed for eligibility and final inclusion of the review

Generalisability Selected studies were predominantly conducted in Australia $(n=10)$, and of that, only two studies sampled participants nation-wide. Several studies were specific to the regions in which the studies were conducted, like Townsville (QLD), Kimberley (WA), Pilbara (WA), and Saskatchewan (Canada). Hence, a Poor (level D) was applied.

Applicability The evaluated evidence was rated Satisfactory (level C). The existing differences in findings, perspectives, and recommendations highlighted in this review should not hinder direct application of studies' findings when considered with Indigenous Australian populations.

The overall body of evidence was at a poor to satisfactory level, which constrains any conclusions that can be drawn about EPDS use and highlights the urgent need for further research in this area. There are several key findings methodological from the current study that can helpfully inform planning for future research.

\section{Key findings}

Psychometric validation In a prospective study, it was felt that congruence between a woman's DSM-IV diagnosis and her high EPDS score was validated by her feelings of disinterest in her baby during pregnancy, not bonding with her child at birth, and feeling overwhelmed during postpartum (Clarke 2008b). Two other studies found that, according to this psychometric validation, the standard EPDS was suitable for use with First Nations and Metis women (Clarke 2008b) and American Indian and Alaska Native (AI/AN) women (Heck 2018). However, instruments like the PDSS and BDI-II were used in these studies to validate the EPDS (Clarke 2008b), yet these have not themselves been validated with Indigenous populations, and cultural differences in preferences, biasses, and interpretations of mental health were noted (Heck 2018). This "chicken and egg" quandary needs to be resolved in Indigenous validation studies - a mixed methodology involving "blind" review by local Indigenous health workers is one potential solution, and inviting mothers and healthcare workers to reflect on their experience of using the EPDS is another.

Perceptions and expressed concerns Three qualitative Australian studies reported views from the HCP's perspective, indicating mixed results. Some found that HCP had low regard for EPDS scores when used with Indigenous women (WAPMHU and WNHS 2011), others found the EPDS was not a meaningful measure due to language barriers (Gausia et al. 2013) and that it was insulting and culturally inappropriate, whilst others felt that it was an acceptable measure (Highet and Goddard 2014). A Queensland-wide survey reported $37 \%$ of clinicians raised concerns about the EPDS not accurately identifying issues and 58\% clinicians reported that the EPDS lacked cultural relevance (Queensland Health 2013). 


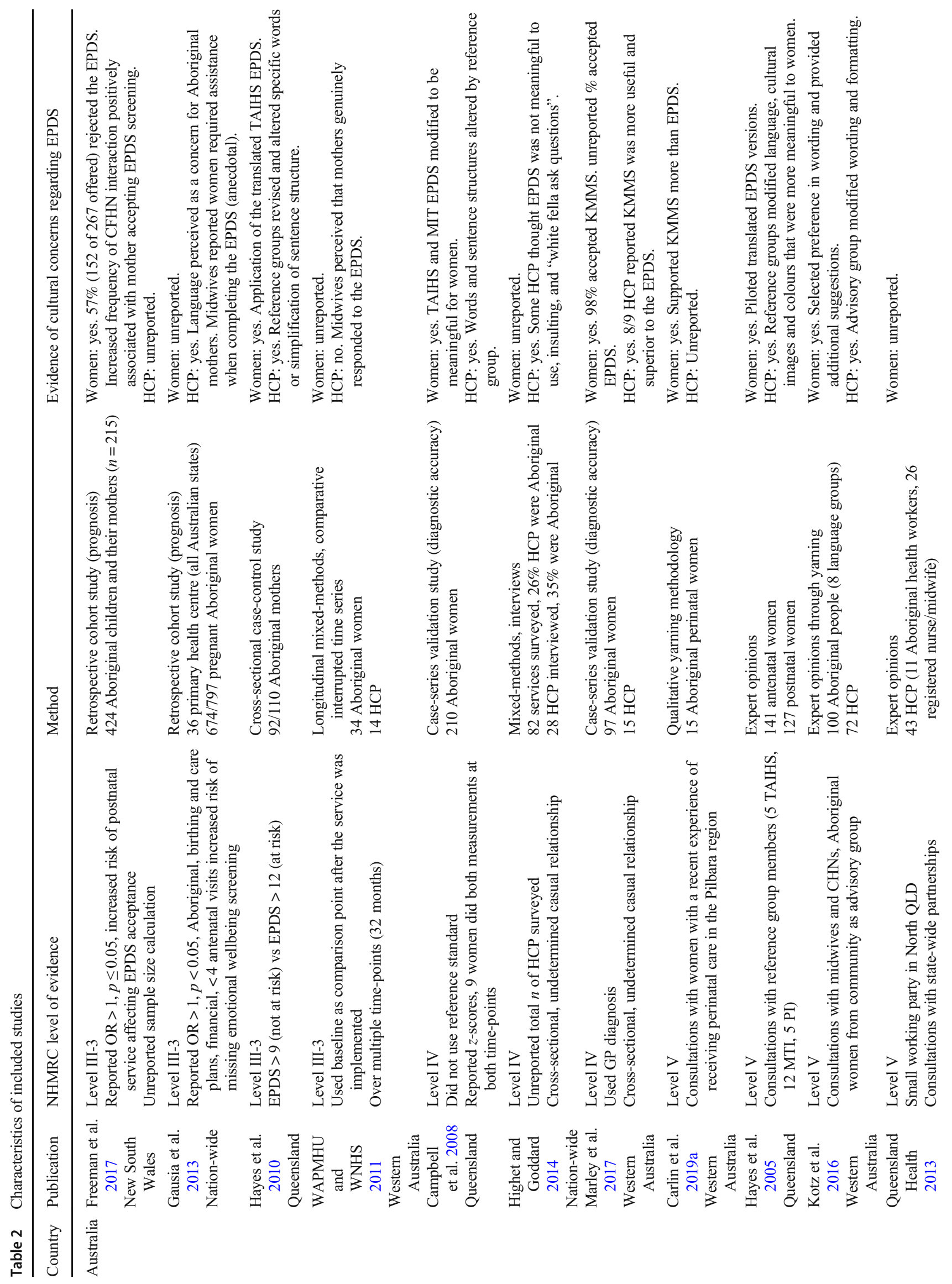




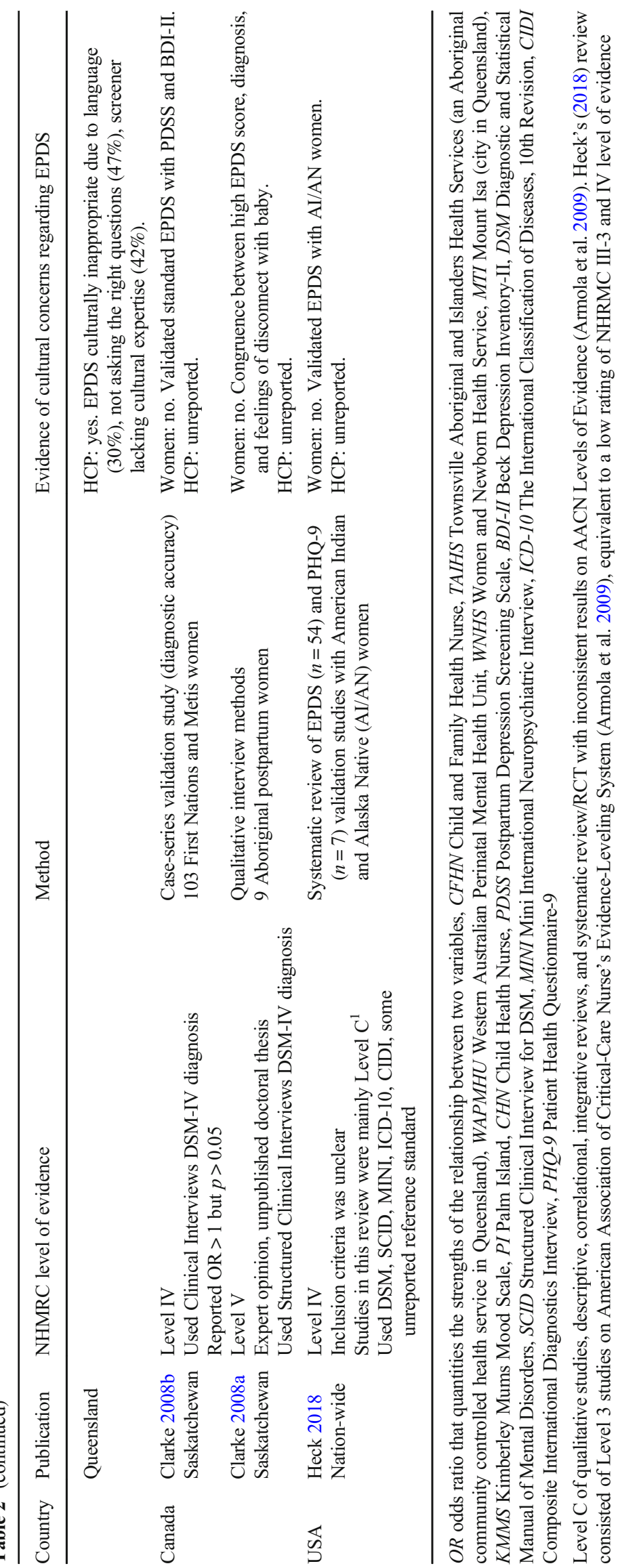




\section{Actions speak louder than words: looking for incidental indicators}

EPDS rejection Taking a population health approach to the issue of cultural validity, one Australian study found that the reported overall rate of women completing the EPDS was lower than those who rejected EPDS screening (43\% vs $57 \%, n=115$ ) and drastically lower than the rate of completion by mothers in the general population $98 \%$ (Freeman et al. 2017). The lack of discussion of underlying reasons for EPDS rejection was a significant design limitation, though a higher EPDS rejection rate is telling in itself. These findings also highlight the potential for biassed sampling in validation studies. Since only a minority of women are turning up for screening, it can be argued that those who do not attend may be a more high-risk sample and critical for validation of the instrument.

Cultural translations and adaptations Cultural concerns around standard EPDS suitability are implicit in the decision to translate the EPDS into the Townsville Aboriginal and Islanders Health Services (TAIHS) version (Campbell et al. 2008; Hayes et al. 2005; Hayes et al. 2010) and adapt the EPDS to form the Kimberley Mums Mood Scale (KMMS) (Carlin et al. 2019a; Kotz et al. 2016; Marley et al. 2017). Perinatal Aboriginal women in the Pilbara region in WA supported the KMMS and preferred it over the EPDS because of its simplified language and yarning element of KMMS (Carlin et al. 2019a).

These modifications and translations address perceived wording and formatting limitations (Kotz et al. 2016) and reflect more implicit and common usage of words in the local dialect (Hayes et al. 2010) as well as the use of face emoticon in place of a numeric Likert scale. Studies using translated or adapted EPDS versions demonstrated an intended movement towards a more culturally safe screening process for Indigenous women and families (Hayes et al. 2010; Marley et al. 2017); however, there have also been some challenges to the validity of these instruments, for example, Hayes et al. (2005) found a response bias in which women persistently selected the happiest emoticon face.

Modified approaches to implementing EPDS A mixedmethods questionnaire revealed that Australian HCPs working with Indigenous mothers described adaptations to the implementation of the EPDS - specifically, using the EPDS in an informal, conversational way (Highet and Goddard 2014). Some HCPs (21\%) weave the EPDS questions into conversation, whilst an alternative approach to EPDS implementation was to provide assistance (Gausia et al. 2013), modify the words to suit the client and to add prompts about family involvement (i.e., partner), and support networks (Highet and Goddard 2014). Part II of the KMMS was designed to address these issues by opening a discussion around additional emotional, social, or physical health concerns or risk factors (Marley et al. 2017). As well as implementing Part II of KMMS, the kinds of questioning and how the HCP approached such sensitive questions were critically important too (Carlin et al. 2019a). Australia's perinatal best practice guidelines currently state that language and cultural appropriateness of the EPDS should be considered when screening with Indigenous women, such as adapting the way the questions are asked (Austin et al. 2017), though it is not clear how this should be achieved.

Culturally relevant research methodology Studies describing the process of tool development (TAIHS, KMMS) outlined the importance of being patient, providing time and space to listen to and seek endorsement from Indigenous women with respect to preferences or suggestions for wording, emerging layout themes, and implementation management and strategies (Campbell et al. 2008; Hayes et al. 2005; Kotz et al. 2016; Marley et al. 2017). Allowing Indigenous women and communities to have a voice is necessary in building strong partnerships with Indigenous communities (Campbell et al. 2008; Carlin et al. 2019a; WAPMHU and WNHS 2011). Possible strategies to enhance community engagement and participation is the use of yarning technique (Carlin et al. 2019a; Kotz et al. 2017) or the use of Indigenous interviewers (Clarke 2008a). Yarning, as a research methodology, follows an open-ended and conversational approach that acknowledges Indigenous principles of oral language, story-telling, and shared experiences (Bernardes et al. 2020; Walker et al. 2014). The method of yarning is essential in the research process as it highlights reciprocity between the participant and researcher and is based around principles of respect, empowerment, and cultural sensitivity (Smith et al. 2020). Qualitative methods, similar yet distinguishable to the yarning style, uncovered rich experiences of using the EPDS that was complementary to the EPDS score itself (Carlin et al. 2019a; Clarke, 2008a; Highet and Goddard 2014; Marley et al. 2017; WAPMHU and WNHS 2011).

\section{Discussion}

The cultural acceptability of the EPDS within an Indigenous context remains unclear and unresolved. Resolution of this issue is a high priority given the disturbingly poor health and wellbeing outcomes for Indigenous mothers and babies. Together, these studies have identified methodological limitations and, thus, suggest a potentially stronger focus on 
methodological design and approaches when addressing cultural security issues in future research.

\section{Questioning current EPDS implementation}

Findings continuously indicate that Indigenous women are being less likely to be screened than non-Indigenous women (Gausia et al. 2013; San Martin Porter et al. 2019), perhaps because Indigenous women may be less likely to attend antenatal appointments (San Martin Porter et al. 2019) or that Indigenous women may be less likely to accept the invitation to complete the EPDS (Freeman et al. 2017). Studies identified barriers to EPDS screening such as issues around fear and stigma (Highet and Goddard 2014), whilst other studies highlighted enablers such as trust, engagement, and ongoing commitment with Indigenous groups (Freeman et al. 2017; Kotz et al. 2016; WAPMHU and WNHS 2011). However, even with the newly developed translations and adaptations of the EPDS, findings of suitability (i.e., cut-off points, validity) were inconclusive. Adaptive approaches to EPDS implementation, like deeper inquiry and further discussions rather than the traditionally structured question-and-answer approach to screening, may have positive implications for engagement but also potentially have negative implications for validation and moreover, for the way the EPDS results are interpreted clinically and in research studies.

Notably, whilst gaining international use in clinical and research work over the years, the EPDS has been used in potentially substandard and misleading ways that departed from the original purpose. The original authors and developers clarified that the EPDS should not be used as a diagnosis assessment, but rather as part of screening process that facilitates "discussing her responses and listening to her story" (Cox 2017, 2019). Considering the low-quality evidence of this current systematic review, researchers and clinicians should understand, refer back, and adhere to the original intent of the EPDS.

Finally, due acknowledgement is required to the unique differences in interpretation and perceptions of emotional mental health and manifestation of postpartum depression and anxiety across Indigenous groups in America (Heck, 2018) and Indigenous Australian populations (Buist et al. 2007; State of Queensland 2014). For instance, "social and emotional wellbeing" (SEWB) is preferred by Indigenous Australians because of its holistic, emotional, and social connotations of health (Gausia et al. 2015). The EPDS fails to consider these differences and can contribute to women answering differently, or to significant aspects of mental wellness not being captured by the tool at all. In light of cultural variations of psychological experiences and cultural norms of mental health conceptualisation, EPDS findings should be interpreted with caution and cultural sensitivity.
Given that neither EPDS nor SEWB measures have been validated with Indigenous Australian groups, it may be that SEWB enquiry can fruitfully supplement EPDS screening for Indigenous Australians women - minimally SEWB inquiry covers well-established risk factors for mental health disorders in Indigenous communities (Gausia et al. 2015). In the absence of a contextually validated measure, more supplementary attention should be paid to the historical, cultural, social, and economic contexts and aspects of mental health.

\section{Critical reflection: acceptability and validation}

Cultural translations, adaptations, and modifications to the standard EPDS have been proposed, developed, and implemented. These may be considered more acceptable perinatal mental health screening practices with Indigenous populations. Currently, acceptability is being prioritised over validation but given that the purpose of perinatal assessment is to use results to guide treatment decisions and resource allocation, both elements of validity should be included together in future studies. Perhaps emphasis could be given to the need for both a culturally acceptable and methodologically robust version of the EPDS to ensure that there is no significant trade-off between these two elements.

Possibly of more importance is that the available studies highlighted the difficulty in developing and evaluating a new measure. There was notably limited evidence of sound psychometric properties of the adapted instruments and little evidence that managed to ascertain whether the EPDS, when used in its standard format, was sensitive to identifying those at risk for perinatal depression in this context. Minimally, improved methodology for adaptations and evaluations warrants exploration and critical reflection.

\section{Mixed-methods research methodology}

Employing culturally relevant research methodologies is a way to honour, respect, and prioritise culture by expressing a genuine partnership with those who are trusted in both cultures (Buist et al. 2007). Gathering stories by yarning is aligned with Indigenous' oral narrative culture and offers great potential for better understanding women's experiences (Lin et al. 2016). However, the qualitative studies presented in this review were not methodologically strong. For instance, the qualitative approach in validating the KMMS shed light on its acceptability compared to the standard EPDS (Carlin et al. 2019a; Marley et al. 2017) but it remains unclear whether this measure provides a safe way of understanding the risk of depression in Indigenous mothers. Encouragingly, a more detailed study protocol has been proposed to evaluate and further confirm validation/revalidation and acceptability of the KMMS in the Kimberley, Pilbara, and far north Queensland regions in Australia (Carlin et al. 2019b). 
Reciprocal cultural consultation and shared cultural wisdom between reference groups and conversations with community-based Indigenous women was key in facilitating trusted relationships and partnerships with the researchers (Campbell et al. 2008; Carlin et al. 2019a; Hayes et al. 2005; Kotz et al. 2016; Marley et al. 2017). There was general agreement that without trusting collaboration and allies, Indigenous participants and communities will continue to view research as culturally unsafe and as another form of loss of control and ownership (Owais et al. 2020). If trust is hard to gain, and could be even harder to gain when quantitative methods are applied, perhaps qualitative methods could be the preferred path. In addition to the inherent strengths of qualitative methods in this context, the resultant relationships may also lay a stronger foundational groundwork for further quantitative (psychometric) process to be introduced at a later time.

Rather than minimising either the quantitative or qualitative methods, a mixed-methods approach integrating both methodological design may ultimately provide a stronger design (Johnson and Onwuegbuzie 2004). The undertaking of a mixed-methods approach that focuses on building rapport and trust with Indigenous women may be more culturally effective in encouraging Indigenous research participation and community engagement. Furthermore, process-oriented documentation and reflective reporting in research should be considered a key form of accountability in order to learn from both successful methodologies, challenges and barriers, and mistakes.

\section{Limitations of included studies}

One significant reason for the poor quality of results may be the variability in implementation of the measures as HCP lacked awareness and knowledge in understanding and interpreting the EPDS (i.e., using it as a diagnostic tool; WAPMHU and WNHS 2011). Many papers suggested that HCP did not adhere to standard protocol when implementing the EPDS, and also possibly, when implementing other measures. An error in EPDS administration or lack of accuracy introduces nonstandardised error variance to case identification using the EPDS tool, questioning the clinical impacts this has on clients as well as undermining interpretation of research findings and collecting accurate health data (Di Florio et al. 2017).

Notably, cultural concerns about the EPDS were derived primarily from perspectives of HCP and not confirmed by women, so inferences about cultural concerns were tentative and not strong in quality. For example, underlying reasons for EPDS rejection were not further explored or discussed in detail (Freeman et al. 2017). Whilst there is reported evidence of Indigenous mothers who have accepted either the EPDS or an alternative measurement/assessment, it remains unclear whether participants avoided screening because of the EPDS as a tool or because of a wider issue regarding stigma of mental health screening in general.

Those that included both perspectives were studies that translated and adapted the standard EPDS. Yet, these studies presented methodological limitations including poor psychometric validation and lack of generalisability. Together, these limitations pose a risk for accuracy of case identification using the EPDS and significantly restricts the confidence in the interpretation of the findings.

Clinical anxiety indicators of the EPDS were not extensively discussed within the international Indigenous literature, and where it was discussed, anxiety disorders were discussed in conjunction with depressive disorders rather than as a standalone feature. The EPDS anxiety subscale (items 3, 4, and 5) has been suggested to screen for anxiety in perinatal women (Bowen et al. 2008; Grigoriadis et al. 2011; Matthey 2008; Matthey et al. 2013). Indigenous women commonly experience anxiety as mothers-to-be and have additional stresses of other complex and multifaceted factors and vulnerabilities that come into play in relation to mental health and wellbeing (Carlin et al. 2019a; Parker and Milroy 2014). To avoid missed opportunities for case identification, future studies could determine a distinction between anxiety and depression when enquiring about psychosocial factors by means of considering the EPDS anxiety subscale as a separate score from the total EPDS score (Jomeen and Martin 2005).

In sum, validation studies remain critically needed in an international context. The absence of culturally valid referent measures remains a challenge-a step-by-step process is required.

\section{Limitations of this review}

This review attempted to include a wider global perspective of the cultural suitability of the EPDS used with Indigenous populations. We found a concerningly limited evidence-base. The majority of included studies focused on Indigenous communities in Australia, with only three of 14 studies from the USA and Canada. There were no available studies that included Indigenous populations in other geographical regions like New Zealand, Europe, Africa, South America, Pacific Islanders, and the Polynesian and Artic countries. This lack of global evidence-base for use of the EPDS with Indigenous communities is a particularly important finding given the ubiquity of use of the EPDS around the world.

The scope of this review focused on the cultural suitability of EPDS with Indigenous women. Studies that simply reported prevalence of perinatal depression or depressive symptoms (Daoud et al. 2019; Nelson et al. 2018; Owais et al. 2020; Stock et al. 2013; Theme Filha et al. 2016) and thoughts of self-harm during pregnancy (Shah et al. 2011) as indicated by the EPDS were not included as cultural views of the EPDS were not discussed. Unexpectedly but equally important, the 
focus of culturally sensitive research methodology was an essential component of this review. For example, a study that triaged mothers who entered the emergency department at a paediatric hospital was not considered a culturally robust and comprehensive methodology to recruit an Indigenous sample where Indigenous women were poorly represented and were recruited by chance (Stock et al. 2013). The EPDS has also been researched with other Indigenous groups like Māori people in New Zealand (Waldie et al. 2015). Studies as such were excluded from the review but could potentially provide evidence of EPDS usability in other settings or contexts that are not within the scope of this review.

As described, studies were also heterogeneous in their design and methodologies were of variable quality making it hard to combine, compare, and synthesise findings. The disunity in findings between countries and within countries may reflect actual differences or may be a methodological artefact. There were studies that reported either from HCP's or women's points of views. Although understanding HCP's views shed light on the research question, hearing from a third person's view of whether the EPDS is deemed suitable for women does not constitute privileged evidence. Triangulation of multiple lines of evidence fosters a strongest inference through a process of looking at points of congruence in evidence (Creswell and Plano Clarke 2018; Thurston et al. 2014).

Finally, whilst there were two raters working both independently and collaboratively on identifying, extracting, and synthesising findings, one rater predominantly took lead on the narrative synthesis of findings, whilst the second rater supported and discussed the work as it progressed. It was unknown whether using a different research team or more reviewers would yield different results (Lucas et al. 2007).

\section{Conclusion}

A review of the literature was carried out to synthesise available evidence on whether the use of a widely accepted perinatal screening instrument (EPDS) is culturally suitable for Indigenous people across the world. Each study had different aims, methodology, and conclusions, and the quality of methodology was poor when considered against the NHMRC criteria, and therefore, results were inconclusive. However, findings highlight some significant concerns and underline a clear clinical urgency for more robust and culturally secure and stable research to ensure that our screening measures are helpful for this at-risk group of mothers.

Acknowledgements The authors thank Nicole Ilich, $\mathrm{PhD}$ candidate, for helpful and valuable advice on data interpretation.
Author's contributions W Chan: project development, data collection, interpretation of data, manuscript writing, revision, and approval of final version.

C Reid: project development, interpretation of data and discussion of results, revision of manuscript, provided critical feedback, and approval of final version.

P Skeffington: project development, planning and data collection, interpretation of data, revision of manuscript, provided critical feedback, and approval of final version.

R Marriott: project development, cultural oversight and supervision, revision of manuscript, provided critical feedback, and approval of final version.

\section{Compliance with ethical standards}

Ethical approval This study does not contain any studies with human participants or animals performed by any of the authors.

Conflict of interest The authors declare that they have no conflict of interest.

Open Access This article is licensed under a Creative Commons Attribution 4.0 International License, which permits use, sharing, adaptation, distribution and reproduction in any medium or format, as long as you give appropriate credit to the original author(s) and the source, provide a link to the Creative Commons licence, and indicate if changes were made. The images or other third party material in this article are included in the article's Creative Commons licence, unless indicated otherwise in a credit line to the material. If material is not included in the article's Creative Commons licence and your intended use is not permitted by statutory regulation or exceeds the permitted use, you will need to obtain permission directly from the copyright holder. To view a copy of this licence, visit http://creativecommons.org/licenses/by/4.0/.

\section{References}

Aguiar W, Halseth R (2015) Aboriginal peoples and historic trauma: the processes of intergenerational transmission. National Collaborating Centre for Aboriginal Health. https://www.ccnsa-nccah.ca/docs/ context/RPT-HistoricTrauma-IntergenTransmission-AguiarHalseth-EN.pdf. Accessed on 28 Dec 2018

Alvarado R, Alvarado R, Jadresic E, Jadresic E, Guajardo V, Guajardo V et al (2015) First validation of a Spanish-translated version of the Edinburgh Postnatal Depression Scale (EPDS) for use in pregnant women. A Chilean study. Arch Womens Ment Health 18(4):607612. https://doi.org/10.1007/s00737-014-0466-z

American Psychiatric Association (2013) Diagnostic and statistical manual of mental disorders, 5th edn. American Psychiatric Association, Washington

Anderson I, Robson B, Connolly M, Al-Yaman F et al (2016) Indigenous and tribal peoples' health (The Lancet-Lowitja Institute Global Collaboration): a population study. Lancet 388:131-157. https:// doi.org/10.1016/S0140-6736(16)00345-7

Armola RR, Bourgault AM, Halm MA, Board RM, Bucher L, Harrington L, Heafey CA, Lee R, Shellner PK, Medina J (2009) AACN levels of evidence: what's new? Crit Care Nurs 29(4):70-73. https://doi. org/10.4037/cen2009969

Austin M-P, Highet N, the Expert Working Group (2017) Mental health care in the perinatal period: Australian Clinical Practice Guideline. Centre of Perinatal Excellence, Flemington https://www.cope.org. au/wp-content/uploads/2018/05/COPE-Perinatal-MH-Guideline Final-2018.pdf. Accessed on 17 Oct 2018 
Australian Institute of Health and Welfare (2014) Birthweight of babies born to indigenous mothers. Australian Institute of Health and Welfare's Indigenous Observatory https://www.aihw.gov.au/ getmedia/a5b1a8e7-ae6a-4f94-8325-926ab446ef04/17597.pdf. aspx ?inline $=$ true. Accessed on 14 Sep 2018

Bernardes CM, Valery PC, Arley BD, Pratt G, Medlin L, Meiklejohn JA (2020) Empowering voice through the creation of a safe space: an experience of aboriginal women in regional Queensland. Int J Environ Res Public Health 17(5):1476. https://doi.org/10.3390/ijerph17051476

Black EB, Toombs MR, Kisely S (2018) The cultural validity of diagnostic psychiatric measures for indigenous Australians: indigenous cultural validation. Aust Psychol 53(5):383-393. https://doi.org/10. 1111/ap. 12335

Bowen A, Bowen R, Maslany G, Muhajarine N (2008) Anxiety in a socially high-risk sample of pregnant women in Canada. Can J Psychiatr 53(7): 435-440. https://doi.org/10.1177/070674370805300708

Brave Heart MYH, Chase J, Elkins J, Altschul DB (2011) Historical trauma among indigenous peoples of the Americas: concepts, research, and clinical considerations. J Psychoactive Drugs 43(4): 282-290. https://doi.org/10.1080/02791072.2011.628913

Buist A, Ellwood D, Brooks J, Milgrom J, Hayes BA, Sved-Williams A, Barnett B, Karatas J, Bilszta J (2007) National program for depression associated with childbirth: the Australian experience. Best Pract Res: Clin Obstet Gynaecol 21(2):193-206. https://doi.org/10.1016/ j.bpobgyn.2006.11.003

Campbell A, Hayes B, Buckby B (2008) Aboriginal and Torres Strait Islander women's experience when interacting with the Edinburgh Postnatal Depression Scale: a brief note. Aust J Rural Health 16(3): 124-131. https://doi.org/10.1111/j.1440-1584.2007.00930.x

Cao-Lei L, de Rooij SR, King S, Matthews SG, Metz GAS, Roseboom TJ, Szyf M (2017) Prenatal stress and epigenetics. Neurosci Biobehav Rev. https://doi.org/10.1016/j.neubiorev.2017.05.016

Carlin E, Atkinson D, Marley JV (2019a) 'Having a Quiet Word': yarning with aboriginal women in the Pilbara region of Western Australia about mental health and mental health screening during the perinatal period. Int J Environ Res Public Health 16(21):4253. https://doi.org/10.3390/ijerph16214253

Carlin E, Blondell SJ, Cadet-James Y, Campbell S, Williams M, Engelke C, Taverner D, Marriott R, Edmonds K, Atkinson D, Marley JV (2019b) Study protocol: a clinical trial for improving mental health screening for Aboriginal and Torres Strait Islander pregnant women and mothers of young children using the Kimberley Mum's Mood Scale. BMC Public Health 19(1):1521-1510. https://doi.org/10. 1186/s12889-019-7845-3

Clarke PJ (2008a) Saskatchewan Aboriginal women's postpartum depressive experiences: a qualitative exploration. Doctoral dissertation, University of Saskatchewan

Clarke PJ (2008b) Validation of two postpartum depression screening scales with a sample of First Nations and Métis women. Can J Nurs Res 40(1):112-125 http://cjnr.archive.mcgill.ca/article/ viewFile/2111/2105

Cook N, Ayers S, Horsch A (2018) Maternal posttraumatic stress disorder during the perinatal period and child outcomes: a systematic review. J Affect Disord 225:18-31. https://doi.org/10.1016/j.jad.2017.07.045

Cox J (2017) Use and misuse of the Edinburgh Postnatal Depression Scale (EPDS): a ten point 'survival analysis'. Arch Womens Ment Health 20(6):789-790. https://doi.org/10.1007/s00737-017-0789-7

Cox J (2019) Thirty years with the Edinburgh Postnatal Depression Scale: voices from the past and recommendations for the future. $\mathrm{Br} \mathrm{J}$ Psychiatry 214(3):127-129. https://doi.org/10.1192/bjp.2018.245

Cox J, Holden J, Henshaw C (2014) Perinatal mental health: the Edinburgh Postnatal Depression Scale (EPDS) manual, 2nd edn. RCPsych publications, London

Cox J, Holden J, Sagovsky R (1987) Detection of postnatal depression: development of the 10-item Edinburgh Postnatal Depression Scale. Br J Psychiatry 150(6):782-786. https://doi.org/10.1192/bjp.150.6.782
Creswell JW, Plano Clark VL (2018) Designing and conducting mixed methods research, 3rd edn. SAGE Publications, Los Angeles

Daoud N, O’Brien K, O'Campo P, Harney S, Harney E, Bebee K et al (2019) Postpartum depression prevalence and risk factors among Indigenous, non-Indigenous and immigrant women in Canada. Can J Public Health 110(4):440-452. https://doi.org/10.17269/s41997-019-00182-8

Department of Health (2019) Clinical practice guidelines: pregnancy care, 2019th edn. Australian Government Department of Health https://beta.health.gov.au/resources/pregnancy-care-guidelines. Accessed on 28 Dec 2019

Department of Health, Government of Western Australia (2006) Edinburgh postnatal depression scale (EPDS): translated versions - validated. State Perinatal Mental Health Reference Group. https://www.mcpapformoms.org/Docs/Edinburgh\% 20Depression $\% 20$ Scale $\% 20$ Translated $\% 20$ Government $\% 20$ of $\%$ 20Western\%20Australia\%20Department\%20of\%20Health.pdf. Accessed 28 Dec 2019

Derrick GE, Hayen A, Chapman S, Haynes AS, Webster BM, Anderson I (2012) A bibliometric analysis of research on indigenous health in Australia, 1972-2008. Aust N Z J Public Health 36(3):269-273. https://doi.org/10.1111/j.1753-6405.2011.00806.x

Di Florio A, Putnam K, Altemus M, Apter G (2017) The impact of education, country, race and ethnicity on the self-report of postpartum depression using the Edinburgh Postnatal Depression Scale. Psychol Med 47(5): 787-799. https://doi.org/10.1017/S0033291716002087

Douglas VK (2013) Introduction to Aboriginal health and health care in Canada: bridging health and healing. Springer, New York

Eastwood J, Ogbo FA, Hendry A, Noble J, Page A, Early Years Research Group (EYRG) et al (2017) The impact of antenatal depression on perinatal outcomes in Australian women. PLoS One 12(1): e0169907. https://doi.org/10.1371/journal.pone.0169907

Freeman J, Murphy E, Woolfenden S, Waters E, Winters J, Callaghan L, Zwi K (2017) Can a child and family health service improve early childhood health outcomes in an urban Aboriginal community?: Aboriginal early childhood health. J Paediatr Child Health 54(5): 541-545. https://doi.org/10.1111/jpc.13785

Gausia K, Thompson S, Nagel T, Rumbold A, Connors C, Matthews V, Boyle J, Schierhout G, Bailie R (2013) Antenatal emotional wellbeing screening in Aboriginal and Torres Strait Islander primary health care services in Australia. Contemp Nurs 46(1):73-82. https://doi.org/10.5172/conu.2013.46.1.73

Gausia K, Thompson SC, Nagel T, Schierhout G, Matthews V, Bailie R (2015) Risk of antenatal psychosocial distress in indigenous women and its management at primary health care centres in Australia. Gen Hosp Psychiatry 37(4):335-339. https://doi.org/10.1016/j. genhosppsych.2015.04.005

Geia LK, West R, Power T (2013) Editorial: addressing perinatal mental health issues for Aboriginal and Torres Strait Island parents and their families: working towards better maternity outcomes. Contemp Nurs 46(1):70-72. https://doi.org/10.5172/conu.2013.46.1.70

Gentile S $(2015,2017)$ Untreated depression during pregnancy: short- and long-term effects in offspring. A Systematic Review Neuroscience 342: 154-66. https://doi.org/10.1016/j.neuroscience.2015.09.001

Gibson J, McKenzie-McHarg K, Shakespeare J, Price J, Gray R (2009) A systematic review of studies validating the Edinburgh Postnatal Depression Scale in antepartum and postpartum women. Acta Psychiatr Scand 119(5):350-364. https://doi.org/10.1111/j.16000447.2009.01363.x

Goodman JH (2019) Perinatal depression and infant mental health. Arch Psychiatr Nurs 33(3):217-224. https://doi.org/10.1016/j.apnu.2019. 01.010

Grigoriadis S, de Camps MD, Barrons E, Bradley L et al (2011) Mood and anxiety disorders in a sample of Canadian perinatal women referred for psychiatric care. Arch Womens Ment Health 14(4): 325-333. https://doi.org/10.1007/s00737-011-0223-5 
Guerin B, Guerin P (2012) Re-thinking mental health for Indigenous Australian communities: communities as context for mental health. Community Dev J 47(4):555-570. https://doi.org/10.1093/cdj/ bss030

Harfield SG, Davy C, McArthur A, Munn Z, Brown A, Brown N (2018) Characteristics of Indigenous primary health care service delivery models: a systematic scoping review. Glob Health 14(1):12-11. https://doi.org/10.1186/s12992-018-0332-2

Hayes B, Geia L, Buckby B, Egan M, McCulley J (2005) Queensland intervention initiative: "Indigenous women's project: report of process and preliminary results". In: The beyondblue national postnatal depression program prevention and early intervention 2001-2005, final report volume II: State-based antenatal intervention initiatives. beyondblue, pp 104-115. https://www.beyondblue.org.au/docs/ default-source/8.-perinatal-documents/bw0076-report-beyondbluenational-research-program-vol2.pdf?sfvrsn=2. Accessed on 17 Oct 2019

Hayes BA, Campbell A, Buckby B, Geia LK, Egan ME (2010) The interface of mental and emotional health and pregnancy in urban Indigenous women: research in progress. Infant Ment Health J 31(3):277-290. https://doi.org/10.1002/imhj.20256

Heck J (2018) Screening for postpartum depression in American Indian/ Alaska native women: a comparison of two instruments. Am Indian Alsk Nativ Ment Health Res 25(2):74-102. https://doi.org/10.5820/ aian.2502.2018.74

Hewitt CE, Gilbody SM, Mann R, Brealey S (2010) Instruments to identify post-natal depression: which methods have been the most extensively validated, in what setting and in which language? Int $\mathrm{J}$ Psychiatry Clin Pract 14(1):72-76. https://doi.org/10.3109/ 13651500903198020

Highet NJ, Goddard AK (2014) Aboriginal and Torres Strait Islander perinatal mental health mapping project: a scoping of current practice surrounding the screening, assessment and management of perinatal mental health across Australia's new directions: Mothers and Baby Service program. Centre of Perinatal Excellence. http://cope. org.au/wp-content/uploads/2013/12/COPE_Mapping-Project_ WEB.pdf. Accessed on 17 Oct 2018

Jackson Pulver L, Haswell MR, Ring I, Waldon J, Clark W, Whetung V et al (2010) Indigenous health: Australia, Canada, Aotearoa, New Zealand and the United States: laying claim to a future that embraces health for us all. World Health Organization, Geneva http://www. who.int/healthsystems/topics/financing/healthreport/IHNo33.pdf. Accessed on 13 May 2019

Johnson RB, Onwuegbuzie AJ (2004) Mixed methods research: a research paradigm whose time has come. Educ Res 33(7):14-26. https://doi.org/10.3102/0013189X033007014

Jomeen J, Martin CR (2005) Confirmation of an occluded anxiety component within the Edinburgh Postnatal Depression Scale (EPDS) during early pregnancy. J Reprod Infant Psychol 23(2):143-154. https://doi.org/10.1080/02646830500129297

Jorm AF, Bourchier SJ, Cvetkovski S, Stewart G (2012) Mental health of Indigenous Australians: a review of findings from community surveys. Med J Aust 196(2):118-121. https://doi.org/10.5694/mja11. 10041

Joshi U, Lyngdoh T, Shidhaye R (2020) Validation of Hindi version of Edinburgh Postnatal Depression Scale as a screening tool for antenatal depression. Asian J Psychiatry 48:101919. https://doi.org/10. 1016/j.ajp.2019.101919

Kotz J, Marriott R, Reid C (2020) The EPDS and Australian indigenous women: a systematic review of the literature. Women Birth. https:// doi.org/10.1016/j.wombi.2020.02.007

Kotz J, Munns A, Marriott R, Marley JV (2016) Perinatal depression and screening among Aboriginal Australians in the Kimberley. Contemp Nurs 52(1):42-58. https://doi.org/10.1080/10376178.2016. 1198710
Kozinszky Z, Dudas RB (2015) Validation studies of the Edinburgh Postnatal Depression Scale for the antenatal period. J Affect Disord 176:95-105. https://doi.org/10.1016/j.jad.2015.01.044

Laungani P (2000) Postnatal depression across cultures: conceptual and methodological considerations. Int J Health Promot Educ 38:86-94. https://doi.org/10.1080/14635240.2000.10806156

Lima F, Shepherd C, Wong J, O'Donnell M, Marriott R (2019) Trends in mental health related contacts among mothers of aboriginal children in Western Australia (1990-2013): a linked data population-based cohort study of over 40000 children. BMJ Open 9(7):e027733. https://doi.org/10.1136/bmjopen-2018-027733

Lin I, Green C, Bessarab D (2016) 'Yarn with me': applying clinical yarning to improve clinician-patient communication in Aboriginal health care. Aust J Prim Health 22(5):377-382. https://doi.org/10. 1071/PY16051

Lucas PJ, Baird J, Arai L, Law C, Roberts HM (2007) Worked examples of alternative methods for the synthesis of qualitative and quantitative research in systematic reviews. BMC Med Res Methodol 7(1): 4. https://doi.org/10.1186/1471-2288-7-4

Marley JV, Kotz J, Engelke C, Williams M, Stephen D, Coutinho S, Trust SK (2017) Validity and acceptability of Kimberley Mum's mood scale to screen for perinatal anxiety and depression in remote Aboriginal health care settings. PLoS One 12(1):e0168969. https:// doi.org/10.1371/journal.pone.0168969

Matthey S (2008) Using the Edinburgh Postnatal Depression Scale to screen for anxiety disorders. Depression and Anxiety 25(11):926931. https://doi.org/10.1002/da.20415

Matthey S, Fisher J, Rowe H (2013) Using the Edinburgh Postnatal Depression Scale to screen for anxiety disorders: conceptual and methodological considerations. J Affect Disord 146(2):224-230. https://doi.org/10.1016/j.jad.2012.09.009

Matthey S, Henshaw C, Elliott S, Barnett B (2006) Variability in use of cut-off scores and formats on the Edinburgh Postnatal Depression Scale - implications for clinical and research practice. Arch Womens Ment Health 9(6):309-315. https://doi.org/10.1007/ s00737-006-0152-x

McCabe-Beane JE, Segre LS, Perkhounkova Y, Stuart S et al (2016) The identification of severity ranges for the Edinburgh Postnatal Depression Scale. J Reprod Infant Psychol 34(3):293-303. https:// doi.org/10.1080/02646838.2016.1141346

McIntyre C, Harris MG, Baxter AJ, Leske S, Diminic S, Gone JP, Hunter E, Whiteford H (2017) Assessing service use for mental health by indigenous populations in Australia, Canada, New Zealand and the United States of America: a rapid review of population surveys. Health Res Policy Syst 15(1):67-17. https://doi.org/10.1186/ s12961-017-0233-5

Milgrom J, Gemmill A (2015) Identifying perinatal depression and anxiety: evidence-based practice in screening, psychosocial assessment and management. Wiley, Hoboken. https://doi.org/10.1002/ 9781118509722

Minority Rights Group International (2016) State of the world's minorities and indigenous peoples 2016: events of 2015. Minority Rights Group International, London https://minorityrights.org/wp-content/ uploads/2016/07/MRG-SWM-2016.pdf. Accessed on 26 May 2019

Moher D, Liberati A, Tetzlaff J, Altman DG (2009) Preferred reporting items for systematic reviews and meta-analyses: the PRISMA statement. PLoS Med 6:e1000097. https://doi.org/10.1371/journal. pmed.1000097

National Health and Medical Research Council (2000) How to use the evidence: assessment and application of scientific evidence. National Health and Medical Research Council https://www. nhmrc.gov.au/sites/default/files/documents/reports/clinical\% 20guidelines/how-to-use-evidence-cp69.pdf. Accessed on 13 May 2019

National Health and Medical Research Council (2009a) A guide to the development, implementation and evaluation of clinical practice 
guidelines. National Health and Medical Research Council https:// www.nhmrc.gov.au/about-us/publications/guide-developmentevaluation-and-implementation-clinical-practice-guidelines\#blockviews-block-file-attachments-content-block-1. Accessed on 13 May 2019

National Health and Medical Research Council (2009b) NHMRC additional levels of evidence and grades for recommendations for developers of guidelines: stage 2 consultation. National Health and Medical Research Council. https://www.mja.com.au/sites/default/ files/NHMRC.levels.of.evidence.2008-09.pdf. Accessed on 13 May 2019

National Institute for Health and Care Excellence (2014) Antenatal and postnatal mental health: clinical management and service guidance. National Institute for Health and Care Excellence, London https:// www.nice.org.uk/guidance/cg192. Accessed on 13 May 2019

Nelson C, Lawford KM, Otterman V, Darling EK (2018) Mental health indicators among pregnant Aboriginal women in Canada: results from the Maternity Experiences Survey. Health Promot Chronic Dis Prev Can 38(7-8):269-276. https://doi.org/10.24095/hpcdp.38. $7 / 8.01$

Oates MR, Cox JL, Neema S, Asten P, Glangeaud-Freudenthal N, Figueiredo B, Gorman LL, Hacking S, Hirst E, Kammerer MH, Klier CM, Seneviratne G, Smith M, Sutter-Dallay AL, Valoriani V, Wickberg B, Yoshida K, TCS-PND Group (2004) Postnatal depression across countries and cultures: a qualitative study. Br J Psychiatry 184(46):s10-s16. https://doi.org/10.1192/bjp.184.46.s10

Owais S, Faltyn M, Johnson AVD, Gabel C, Downey B, Kates N, van Lieshout RJ (2020) The perinatal mental health of Indigenous women: a systematic review and meta-analysis. Can J Psychiatr 65:149 163. https://doi.org/10.1177/0706743719877029

Parker R, Milroy H (2014) Mental illness in Aboriginal and Torres Strait Islander peoples. In: Dudgeon P, Milroy H, Walker R (eds) Working together: Aboriginal and Torres Strait Islander mental health and wellbeing principles and practice, 2 nd edn. Commonwealth of Australia, pp 113-124 https://www.telethonkids.org.au/ globalassets/media/documents/aboriginal-health/working-togethersecond-edition/wt-part-2-chapt-7-final.pdf

Peters MA, Mika CT (2017) Aborigine, Indian, Indigenous or First Nations? Educ Philos Theory 49(13):1229-1234. https://doi.org/ $10.1080 / 00131857.2017 .1279879$

Queensland Health (2013) Aboriginal and Torres Strait Islander health workforce capacity building perinatal social and emotional wellbeing project: Phase I report. Queensland Health, Queensland Government, Brisbane https://www.health.qld.gov.au/ data/ assets/pdf_file/0025/439018/smncn-atsi-report-phase1.pdf. Accessed on 14 Sep 2019

Rhee Y, Park J, Cha H, Kim K (2018) The psychometric validation of the EPDS-K among Korean women: does it only measure depressive symptoms? Perspect Psychiatr Care 54(2):115-125. https://doi.org/ 10.1111/ppc. 12207

San Martin Porter MA, Betts K, Kisely S, Pecoraro G, Alati R (2019) Screening for perinatal depression and predictors of underscreening: findings of the born in Queensland study. Med J Aust 210(1):32-37. https://doi.org/10.5694/mja2.12030

Shah SMA, Bowen A, Afridi I, Nowshad G, Muhajarine N (2011) Prevalence of antenatal depression: comparison between Pakistani and Canadian women. J Pak Med Assoc 61(3):242-246

Shrestha SD, Pradhan R, Tran TD, Gualano RC, Fisher JRW (2016) Reliability and validity of the Edinburgh Postnatal Depression Scale (EPDS) for detecting perinatal common mental disorders (PCMDs) among women in low-and lower-middle-income countries: a systematic review. BMC Pregnancy and Childbirth 16(1): 72. https://doi.org/10.1186/s12884-016-0859-2
Smith RL, Devine S, Preston R (2020) Recommended methodologies to determine Australian Indigenous community members' perceptions of their health needs: a literature review. Aust J Prim Health 26(2): 95. https://doi.org/10.1071/PY19078

State of Queensland (2014) Perinatal social and emotional wellbeing screening: a learning package for perinatal psychosocial screening with Aboriginal and Torres Strait Islander peoples. State of Queensland (Queensland Health), Brisbane https://www.health. qld.gov.au/_data/assets/pdf_file/0024/439017/smncn-atsi-reportphase2.pdf. Accessed on 14 Sep 2019

Stock A, Chin L, Babl FE, Bevan CA, Donath S, Jordan B (2013) Postnatal depression in mothers bringing infants to the emergency department. Arch Dis Child 98(1):36-40. https://doi.org/10.1136/ archdischild-2012-302679

Theme Filha MM, Ayers S, Gama SGN, Leal MC (2016) Factors associated with postpartum depressive symptomatology in Brazil: the birth in Brazil National Research Study, 2011/2012. J Affect Disord 194:159-167. https://doi.org/10.1016/j.jad.2016.01.020

Thurston WE, Cove L, Meadows LM (2014) Methodological congruence in complex and collaborative mixed method studies. Int J Mult Res Approaches 2:2-14. https://doi.org/10.5172/mra.455.2.1.2

Trovato F, Romaniuk A (2014) Aboriginal populations: social, demographic, and epidemiological perspectives. The University of Alberta Press, Alberta

United Nations (2014) Permanent forum on indigenous issues: report on the Thirteenth Session (12-23 May 2014), Supplement no. 23. United Nations, New York. http://undocs.org/E/2014/43. Accessed on 26 May 2019

Waddington H, White H, Snilstveit B, Hombrados JG, Vojtkova M, Davies P, Bhavsar A, Eyers J, Koehlmoos TP, Petticrew M, Valentine JC, Tugwell P (2012) How to do a good systematic review of effects in international development: a tool kit. J Dev Eff 4:359 387. https://doi.org/10.1080/19439342.2012.711765

Waldie KE, Peterson ER, D'Souza S, Underwood L, Pryor JE, Carr PA, Grant C, Morton SMB (2015) Depression symptoms during pregnancy: evidence from growing up in New Zealand. J Affect Disord 186:66-73. https://doi.org/10.1016/j.jad.2015.06.009

Walker M, Fredericks B, Mills K, Anderson D (2014) "Yarning" as a method for community-based health research with Indigenous women: the Indigenous women's wellness research program. Health Care for Women Int 35(10):1216-1226. https://doi.org/10.1080/ 07399332.2013.815754

Western Australian Perinatal Mental Health Unit (WAPMHU), Women and Newborn Health Service (WNHS) (2011) Aboriginal perinatal mental health service expansion: final evaluation. Department of Health, State of Western Australia. https://www.kemh.health.wa. gov.au/ /media/Files/Hospitals/WNHS/Our\%20Services/Statewide\%20Services/WHSP/Aboriginal_Final_Evaluation_Report. pdf. Accessed 17 Oct 2018

World Health Organization (2013) Mental Health action plan 2013-2020. World Health Organization, Geneva https://apps.who.int/iris/ bitstream/handle/10665/89966/9789241506021 eng.pdf; jsessionid $=7921375663 \mathrm{~B} 6 \mathrm{~A} 210415 \mathrm{~B} 381942 \mathrm{~A} 58 \mathrm{C} 8 \mathrm{D}$ ? sequence $=$ 1. Accessed on 26 May 2019

World Health Organization (2016) The WHO application of ICD-10 to deaths during the perinatal period: ICD-PM. World Health Organization, Geneva http://apps.who.int/iris/bitstream/handle/ $10665 / 249515 / 9789241549752$-eng.pdf?sequence $=1$. Accessed on 26 May 2019

Publisher's note Springer Nature remains neutral with regard to jurisdictional claims in published maps and institutional affiliations. 